\title{
Características inmunológicas de una población infantil no respondedora a la vacuna $\mathrm{BCG} *$
}

\author{
Benito González M..$^{\mathrm{I}}$; Jaime Lnostroza S. ${ }^{2}$; \\ Ricardo Sepúlyeda $\mathbf{M}^{3}$ \\ Immune responses among BCG vaccinated \\ tuberculin negative infants
}

\begin{abstract}
In vitro immıne responses to Bacillus Camete Guerin (BCG) vaccination were studied in three month old Infants vaccinsted at birth which showed positwe in; 16) or negative in: 16] PPD skin reactions and in non vaccinated infants of the same age $(n \cdot 15)$ by specific PPD induced blastogenesis assay and non specific phytohemaglutinin (PHA) stimulation. In non vaccinated infants blastogenic respenses (cpm) at PPD doses of 25, 50 and $100 \mathrm{Hg} \cdot \mathrm{kere}$ slight 1770 to $1700 \mathrm{coml}$, wrereas among vaccınatec PPO positive intants corresponding values were $27925 \pm$ $7095,34297 \pm 8717$ and $45354 \pm 8453 \mathrm{cpm}$ respectively $p<0.001$ \% . In vaccinated infants showing negative PPD skin reactions, responses were similar to those seen among non vaccinated babies in ten cases, while the other six responded just like vaccinated children with positive PPD reaction $(2744 \pm 7145$ com $)$, but only $\lrcorner$ nder 100 $\mu_{g}$ PPD doses (p $>0.05$ ). Stimulation indexes under PPD or BCG were in all similar. PHA response was 124945 $\pm 53200 \mathrm{cpm}$ in non vaccinated infants. $156263 \pm 43540$ in PPD non responder vaccinateo subjects and $138217 \pm$ 22345 in PPD-positive vaccinated babies $(p>0.05$ ). Stimulation indexes after supressor adherent cell elimination were lower in all studied groups but reached statistical significance only among vaccinated PPD non responders (p < 0.01$)$. Serum concentration of $\mathrm{IgG}$ and IgV antiPPD antibodies were similar among infants of all three groups and in pooled sera from blood bank's healthy donors, but signiticantly lower than in serum from patients with active tuberculous disease.
\end{abstract}

(Key words: Tuberculosis, immunization, BCG vaccine, immunity, cellular, $T$ Iymphoryte, humoral, antiPPD antibodies.]

* Estos estudios se han llevado a cabo con el aporte de un proyecto Fondecyt $1226 / 90$ y por un Grant Thrasher Research Found. Salt Lake City, Utah, USA.
1. Unidad de Inmunologia, Hospital Lujs Calvo Mackenna.

2. Laboratorio de Inmunología, Hospital Regional, Temuco.

3. Servicio de Inmunologia, Hospital INERyCT. 
La utilización de una vacuna para combatir la tuberculosis data desde 1921 , cuando se introdujo por primera vez una cepa atenuada de Mycobacterium bovis. Desde entonces, 182 países han incorporado este procedimiento de inmunización, preferentemente en el periodo de recién nacido, sin que hasta la fecha se conozcan los fundamentos de su eficacia $y$ los cambios que induce en el sistema inmune. Algunos han demostrado la utilidad de la vacuna en la prevención de las formas diseminadas de la enfermedad, pero persisten enormes interrogantes sobre su eficacia en otras localizaciones, especialmente la pulmonar ${ }^{1}$. Dado que el efecto protector de esta vacuna ha variado de $0 \%$ a $80 \%$, la Organización Mundial de la Salud (OMS) recomendó continuar las investigaciones en este campo, considerando las nuevas técnicas inmunológicas disponibles desde hace algunos años ${ }^{2}$.

Las investigaciones sobre las modifiçaciones inmunológicas causadas por e] BCG son escasas y adolecen de serios problemas metodológicos. En efecto, diferentes autores, empleando reacción tuberculínica, describen porcentajes muy variables de hipersensibilidad retardada. Los examenes in vitro no han mostrado correlaciones importantes con determinados factores, pero las poblaciones analizadas y los métodos de análisis han sido muy heterogéneos ${ }^{3}$. Los estudios de poblaciones han arrojado resultados des. alentadores, como ocurrió en la India. Las explicaciones para estos hallazgos se han centrado en la calidad de la vacuna, método de administración $\mathbf{e}$ infecciones ambientales ${ }^{4}$.

La influencia de las técnicas de administración de la vacuna y de algunas variables adqujridas fueron estudjadas por un gnupo de investjgadores nacionales, demostrando que estos factores no tendrian la relevancia que inicialmente se les atribuyó ${ }^{5-7}$. Estos estudios y otros de diferentes paises han concluido que en cierto porcentaje de niños no se logra su sensibilización a esta vacuna, aun cuando se empleen métodos estándar de administración y calidad uniforme de los inóculos. No existen estudios recientes que expliquen esta falta de respuesta, si bien varios investigadores postulan un defecto a nivel genético. Mejores resultados se han obtenido en el análisis de la anergia tuberculínica en pacientes portadores de TBC activa, 20 a $30 \%$ de los cuales muestran reacciones negativas que se correlacionan positivamente con la gravedad del proce$\mathrm{so}^{8,9}$. Dos mecanismos importantes parecen con- dicionar este fenómeno: la activación de mono. citos (células adherentes) con propiedades supresoras y la de linfocitos con receptores $\mathrm{Fc}^{+}$, que también se comportarian como inhibidores de la respuesta inmune. Este estado de supresión inmunológica se acompaña habitualmente de elevación de los títulos de anticuerpos anti-BCG, reflejando probablemente una estimulación de vías altemas de los mecanismos de defensa ${ }^{10}$.

El propósito de este estudio ha sido caracterizar la respuesta inmune de los lactantes vacurados con BCG al nacer, comparándola con la de niños que no recibieron la vacuna. Determinar $\mathrm{si}$, en los niños que no mostraron respuesta in vivo a repetidas dosis de PPD intradérmico, existe evidencia de supresión de inmunidad causada por células adherentes (monocitos) o por anticuerpos bloqueantes, ambos mecanismos descritos en la anergia tuberculosa. La caracterización de estos mecanismos reviste enorme trascendencia, pues permitiria explicar en parte algunos de los fenómenos biológicos que produce el BCG y comprender los motivos por los cuales aún es posible encontrar fracasos de la vacuna en niños que la reciben adecuadamente.

\section{Material y Método}

Selección de pacientes. Los lactantes fueron seleccionados aprovechando una investigación que se lleva a cabo en nuestro pais, auspiciada por la Fundación Thtasher, en donde se evalúan diferentes variables pre y postnatales sobre la respuesta tuberculínica. En el año 1990 se habia acumulado en ese estudio un total de 840 lactantes que habían sido vacunados al nacer y 120 a quienes se postergó la vacunación con BCG hasta que cumplieran 12 meses de edad. Al tercer mes se aplicaron dos dosis intradénmicas de PPD 5 Unidades. separadas por un intervalo de 15 días, a todos los nữos y la reacción fue leída por un personal entrenado. En $10 \%$ de los lactantes vacunados no se encontro indura. ción dérmica después de la prìnera dosis de PPD, por. centaje que se redujo a 7 \% después de la segunda. Estos uiltimos niños fueron considerados no respondedores para los efectos de este estudio y, entre ellos, se seleccionaron por azar 16 lactantes, para constituir el primer grupo. El segundo grupo de este protocolo fue formado con 16 lactantes vacunados al nacer, cuya reacción de PPD incluyó induraciones de 10 o más mm. El tercer grupo, que sirvió de control, estaba constituido por 15 lactantes de 3 meses de edad que no habían sido vacu. nados en el período de recién nacido. Todos los lactantes estaban recibiendo exclusivamente leche materna on el momento del estudio, salvo custro que, además, reclbian relleno con leche artificial; en ninguno se encontraron antecedentes de tuberculosis entre miembros de la familia o de contacto con portadores de la enfermedad. Los 32 lictantes vacunados al nacer y some- 
tidos al estudio presentaban cicatriz visible al momento del estudio. En todos los lactantes anajizados se informó a los padres del objetivo del esbudio, obteniéndose el consentimiento escrito para el ingreso a la investigación.

Evaluación inmunológica. Para analizar la respuestg inmune específica se empleó la prueba de transformación linfoblástica, que estudia la capacidad de respuesta de los linfocitos $\mathrm{T}$ at ser estimulados por un ant ígeno proteico derivado del $M$. ruberculosis (PPD). La inmunocompetencia global se evaluó midiendo la respuesta de los linfocitos a la estimulación con un mitógeno, la fitohemaglutinina.

La prueba de transformación linfoblástica se realizó extrayendo $6 \mathrm{ml}$ de sangre, de la que separaron los linfocitos a través de una gradiente de Ficoll-Hypaque, según el método de Boyum ${ }^{12}$. Las células obtenidas se cultivaron en medio de cultivo especia! RPMl-1640, suplementado con antibióticos. De la suspensión resultante se tomó $0,1 \mathrm{ml}$, conteriendo $1,5 \times 10^{\prime}$ células, que fueron incubadas en cada pocillo de mjcroplacas recubiertas con distintas concentraciones de PPD durante 5 días a $37^{\circ} \mathrm{C}$ y en una atmósfera de $5 \%$ de $\mathrm{CO}_{2}$ en estufa de cultivo celular. Dieciocho horas antes de terminar la incubación se agregó una solución estéril de 3-metil-timidina* $(5,0 \mathrm{Cj} / \mathrm{mmo})$, Amersham, Arlington). Las células fueron cosechadas en papel filtro especial y el contenido del isótopo incorporado por las células estimuladas con PPD se midió por espectrofotometría líquida de centelleo en un contador beta. Las muestras se procesaron por triplicado $y$ el resultado se expresó en términos del promedio de cuentas por minuto (cpm) y la desviación estándar de ellas, ung vez restadas las cuentas basales (sin est ímulo).

En los tres grupos de nir̂́os se estudiaron también los efectos que se producen en la transformación linfobléstica, cuando se depletan de monocitos los cultivos. Para este efecto, alícuotas celulares conteniendo linfocitos y monocitos fueron cultivadas en placas de Petri plásticas, con el fin de obtener la separación de las células monocitarias, adherentes, siguiendo el método de Crowle ${ }^{12}$. De esta manera se pueden efectuat análisis tanto en muestras que contienen linfocitos y monocitos, as í como en muestras sin monicotos.

Con el fin de determinar si la respuesta inmune dirjgida contra el PPD guarda alguna relación con los determinantes antigénicos del BCG, se analizaron muestras de los tres grupos en la prueba de transformación bléstica, agregando, simultáneamente con el antígeno proteico (PPD), varias dilucione de BCG. La actividad de posibles factores séricos sobre la blastogénesis se evaluó cuantificando los anticuerpos anti-PPD $y$, además, observando el efecto producióo en los cultivos por el suero autólogo y suero heterólogo obtenido de donantes sanos.

La capacidad de respuesta inespecífica que ptesentan los linfocitos $T$ tue determinada por la magnitud de la reacción a un mitógeno, en este caso fítohenaglutinina (PHA), para to cual se cultivaron células mononucleares $(1 \times 10 \mathrm{ml})$ en microplacas con PHA (PHA-M, DIFCO) y RPML 1640 con $10 \%$ de suero humano de grupo sangu íneo $O$ inactivado. Debido a que en nuestro país este método no estaba estandarizado en nifros, se efectuanon diferentes estudios para identificar la concentración óptima de PHA, concluyéndose en que con 10 ug se obtenía buena correlación de dosis y respuesta. Como controles se hicieron cultivos con y sin PHA. Todos los cultivos y los controles se llevaron a cabo en triplicado y fueton incubados a $37 \mathrm{o}^{\circ} \mathrm{C}$ en una atmósfera húmeda de $\mathrm{CO}_{2}$. A las $72 \mathrm{~h}$ de incubación se agregaron $2 \mu \mathrm{Ci}$ de timidina tritiada, luego las células fueron cosechadas sobre papel filtro expecial y el grado de respuesta a la estimulación se calculó a partir de las cuentas obtenidas en un contador de radiación beta's.

La técnica para determinación de anticuerpos anti-PPD ha sido estandarizada y publicada por uno de los autores ${ }^{14}$. Mediante una modificación del método de Kalish se realizó un ensayo inmunoenzimático (ELISA) en placas recubiertas con 10 diluciones diferentes para PPD (Emulon. Dynatech. Alexandria. Virginia. EUA). Estas placas se mantuvieron a $4^{\circ} \mathrm{C}$ durante una noche, luego fueron lavadas 6 veces, bloqueadas con albúmina humana al $5 \%$. A contüuación se agregaron $200 \mu 1$ de suero a cada pocillo y se incubaron por 2 horas a $37^{\circ} \mathrm{C}$. Con el fin de identificar los anticuerpos de origen materno (IgC) y los del propio niño (IgM), se lavaron las preparaciones y se combinaron con antiIgG o anti-lgM humana marcadas con fosfatasa alcalina (Bionetic-Litton, EUA). Posteriormente se adicionó un sustrato consistento en p-nitro-fenilfosfato (Boehtinger Mannheirn). Las muestras se leen en un espectofotometro a una densidad óptica de $405 \mathrm{~nm}$. Con el fin de evaluar el método empleado en la detección de estos anticueтpos, se analizaron concomitantemente 12 muestras provenientes de pacientes portadores de TBC activa y 12 muestras de donantes sanos que concurrieron a un banco de sangre. Las muestras de suero fueron individualizada mediante un código (número), con lo cual el investigador no tuvo acceso a la información hasta no haber entregado los resultados individuales de las muestras.

Los valores obtenidos en los diferentes grupos de niños en el ensayo de blastogénesis se analizaron mediante prueba de varianza de una vía, en tanto los promedios individuales fueron analizados mediante la prueba de Student-Newman-Keuls. El efecto observado en las muestras, después de eliminar las células adherentes o monocisos, fue estudiado mediante la prueba de Student para muestras pareadas ${ }^{1 s}$.

\section{Resultados}

Los cambios experimentados por los linfocitos $\mathrm{T}$ bajo la acción de la vacuna $\mathrm{BCG}$ se demuestran en la figura 1. En las células de lactantes no vacunados se observó escasa respuesta a la estimulación a las diferentes concentraciones de PPD, obteniéndose valores promedios que fluctuaron entre 700 y $1700 \mathrm{cpm}$, mientras en $\mathrm{los}$ niños vacunados con reacción positiva al PPD se registraron cifras muy elevadas de proliferación celular, en estrecha correlación con la dosis de antígeno empleado, siendo las respectivas cPm: $27925 \pm 7095$ para $25 \mu \mathrm{g}$ de PPD; 34297 


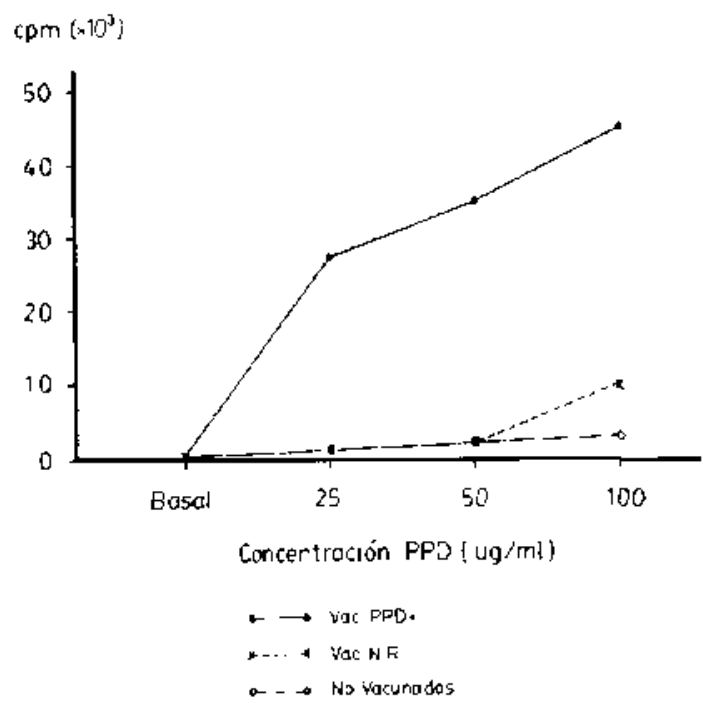

Figura 1: Respuesta proliferativa de linfocitos frente a diferentes concentraciones de PPD en lactantes de 3 meses de edad.

\pm 8717 para $50 \mu \mathrm{g}$, y para $100 \mu \mathrm{g}, 45354 \pm$ $8453 \mathrm{cpm}$. La comprobación estadística mostró que las diferencias entre vacunados y no vacunados eran significativas en las distintas concentraciones de antígeno utilizado $(p<0,05)$. En los niños no respondedores se observó un patrón de respuesta interesante. En efecto, tal como se aprecia en la figura 1 , los indices respuesta a la estimulación con las concentraciones bajas de PPD ( 25 y $50 \mu g$ ) eran semejantes a los de niflos no vacunados $(p>0,05)$. Sin embargo, frente a concentraciones altas de antígeno $(100 \mu \mathrm{g})$ la respuesta fue mayor, del orden de $9635 \pm 3661 \mathrm{cpm}$, similar a la observada en el grupo vacunado $(\mathrm{p}>0,05)$. En el análisis más detallado de estos lactantes se encontró 10 niños con respuestas exactamente iguales a los no vacunados y otros 6 en donde se produjo una ele. vación significativa de la blastogénesis frente a las dosis de $100 \mu \mathrm{g}$ de PPD, con valores promedios de $22744 \pm 7145 \mathrm{cpm}$. El análisis estadístico de este último subgrupo no mostró diferencias con los nifios vacunados PPD positivos ( $\mathrm{p}>0,05$ ) pero sí con los no vacunados y los vacunados no respondedores con recuentos bajos $(\mathrm{p}<0,05)$ (figura 2).

Mediante un análisis de correlación simple estudiamos el grado de transformación blastica que experimentaron las células de 10 nifios vacunados cuando fueron expuestas en los cultivos

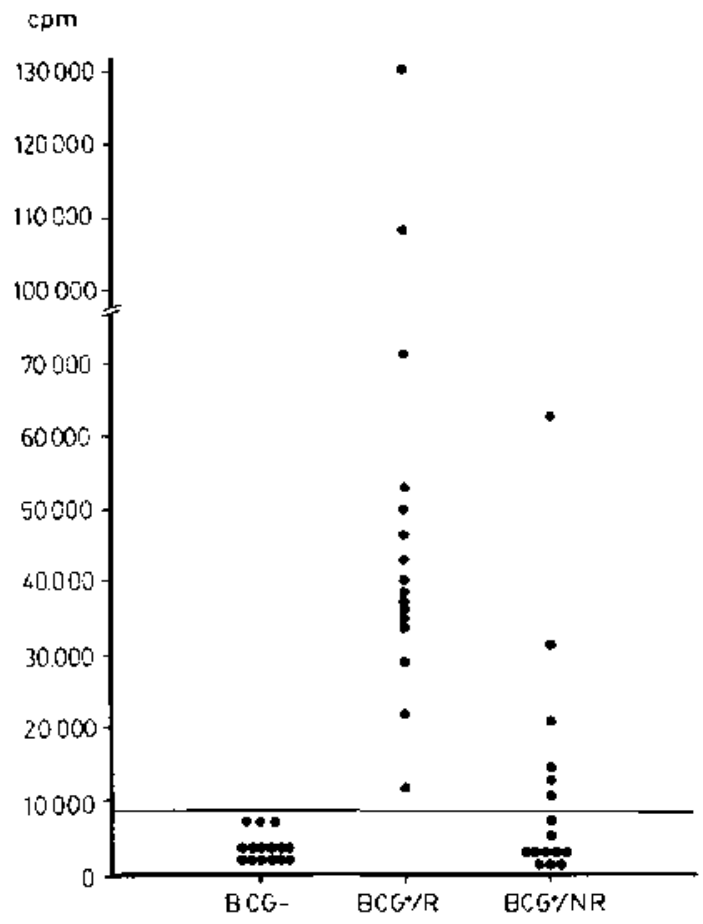

Figura 2: Respuesta proliferativa frente a dosis elevada de PPD (100 ug/ml) de linfocitos procedentes de niños no vacunados (BCG-), vacunados con buena respuesta al $\mathrm{PPD}(\mathrm{BCG}+/ \mathrm{R})$ y niños vacunados no respondedores al PPD (BCG+/NR).

al PPD y a diferentes diluciones de BCG, siendo $\mathbf{r}=0.82(\mathrm{p}<0,01)$, con lo cual se estableció que ambas respuestas presentaron una estrecha homología y no difirieron significativamente en. tre si.

Con el fin de determinar si ciertos factores presentes en el suero de estos niños estuvieran influyendo en las respuestas blastogénicas, se tomaron muestras de 12 lactantes (4 de cada grupo) para incubarlas en presencia tanto de suero autólogo como heterólogo y luego someterlas a cultivos estimulados con distintas concentraciones de PPD y $B C G$, sin que se encontraran diferencias en las respuestas de los cultivos frente a uno u otro tipo de suero.

Cuando analizamos el efecto de la depleción de células adherentes (monocitos) sobre el grado de estimulación linfocitaria, pudimos observar que la eliminación de estas células en los cultivos produjo una disminución importante en el número de cpm en todos los grupos estudiados y a las distintas concentraciones de antígeno em- 
pleado. Sin embargo, en las muestras de uno de los niños del grupo no respondedor ocurrió una elevación estadísticamente significativa de las cuentas cuando se retiraron estas células, desde $8096 \mathrm{cpm}$ a $100 \mu \mathrm{g}$ de PPD, en cultivos con células totales, a $90239 \mathrm{cpm}$ después de retirar los monocitos $(p<0,001)$.

El grupo no respondedor, aun cuando fue el único grupo con un niño que incrementó sus lecturas, fue el más vulnerable a la depleción de monocitos. En efecto, la comparación estadística demostró que a $100 \mu \mathrm{g}$ de PPD, solamente estos ninos tuvieron modificaciones estadisticamente significativas $(\mathrm{p}<0,01)$ cuando se compararon los resultados antes y después de retiradas estas células adherentes. La dismjnución en los indices de estimulación no fue tan marcada para los niños de los demás grupos $(\mathrm{p}>0,05)$.

En la tabla 1 se describen las concentraciones de anticuerpos anti-PPD de clase IgG en el suero de los tres grupos de niños, comparados con sueros provenientes de pacientes con tuberculosis activa y donantes "sanos" de un banco de sangre. Los niveles de $\lg G$ anti-PPD, que pudiera haber sido transferida por vía placentaria o sintetizada por efecto de infección por el $\mathrm{MyCo}^{-}$ bacterium tuberculosis, no mostraron diferencias significativas entre los distintos grupos, salvo en los pacientes con tuberculosis activa, que tenían concentraciones altas IgG anti-PPD. La concentración de anticuerpos era semejante en niños vacunados y no vacunados $(p>0,05)$.
Los niveles de anticuerpos IgM específicos para el bacilo de Calmette-Guérin de la vacuna BCG se comportaron de manera semejante a los anteriores (tabla 2), siendo posible encontras aumentos estadísticamente significativos en pacientes con $\mathrm{TBC}$, frente a todos los grupos y a las diferentes concentraciones de PPD analizadas. Los donantes sanos de un banco de sangre no mostraron diferencias con los niños vacuna. dos y no vacunados $(\mathrm{p}>0,08)$.

El grado de inmunocompetencia celular, medido por la acción de la fitohemaglutinina (PHA), mostró valores promedio, para el grupo no vacunado, de $124945 \pm 53200 \mathrm{cpm}$; para los vacunados no respondedores $156263 \pm$ 43540 ; para los vacunados con PPD positivos $138217 \pm 22345$. Estos valores no demostraron diferencias significativas entre sí $(p>0,05)$.

\section{Discusión}

La vacuna BCG se ha constituido nuevamente en materia de interés científico debido a los adelantos que han experimentado la inmunología y la inmunogenética. E1 desconocimiento de los mecanismos de protección, la aparición de casos de tuberculosis en sujetos vacunados, el aumento de las infecciones tuberculosas entre enfermos HIV positivos, la duración del efecto protector $y$ el valor de las campafias sanitarias de prevención y pesquisas forman parte de una serie de interrogantes que la medicina deberá contestar

Tabla 1

Concentración de anticuerpos IgG anti-PPD medidos por inmunoanálisis ligado a enzimas

\begin{tabular}{|c|c|c|c|c|c|c|c|}
\hline \multirow[b]{2}{*}{ Grupos } & \multicolumn{7}{|c|}{ Concentraciones de PPD } \\
\hline & $1 / 20$ & $1 / 40$ & $1 / 80$ & $1 / 160$ & $1 / 320$ & $1 / 640$ & $1 / 1280$ \\
\hline No vac. & 1,7719 & 1,6660 & 1,5526 & 1,4178 & 1,3063 & 1,1405 & 0,9476 \\
\hline Vac. NR. & 1,7521 & 1,6501 & 1,5663 & 1,4658 & 1,3591 & 1,2267 & 1,0349 \\
\hline Vac. Re. & 1,5774 & 1,4868 & 1,3974 & 1,2852 & 1,2003 & 1,0082 & 0,8081 \\
\hline TBC & 1,9090 & 1,8810 & $1,9570^{*}$ & $1,8240^{*}$ & $1,7689^{*}$ & $1,6390^{*}$ & $1,4470^{*}$ \\
\hline Control & 1,6510 & 1.5730 & 1,4290 & 1,2904 & 1,1920 & 1,0570 & 0,7560 \\
\hline
\end{tabular}

$\begin{array}{lll}\text { Novac. } & = & \text { no vacunados }(15) \\ \text { Vac. NR. } & = & \text { vacunados no respondedores al PPD (16). } \\ \text { Vac. Re. } & = & \text { vacunados con PPD positivos (16). } \\ \text { TBC } & = & \text { pacientes con TBC activa (12) } \\ \text { Control } & = & \text { donantes de banco de sangre (12). } \\ & \text { * }<<0,05 .\end{array}$


Tabla 2

Concentración de anticuerpos IgM anti-PPD medidos por inununoanálisis ligado a enzimas

\begin{tabular}{|c|c|c|c|c|c|c|c|}
\hline \multirow[b]{2}{*}{ Grupos } & \multicolumn{7}{|c|}{ Concentraciones de PPD } \\
\hline & $1 / 20$ & $1 / 40$ & $1 / 80$ & $1 / 160$ & $1 / 320$ & $1 / 640$ & $1 / 12 B 0$ \\
\hline No vac. & 0,6625 & 0,5758 & 0.5085 & 0,4426 & 0,3876 & 0,3276 & 0,2706 \\
\hline Vac. NR. & 1,0974 & 0,9533 & 0,8488 & 0,7317 & 0,6221 & 0,4099 & 0,3820 \\
\hline Vac. Re. & 0,8544 & 0,7392 & 0,6344 & 0,5263 & 0,4477 & 0,3667 & 0,3008 \\
\hline TBC & $1,8160^{*}$ & $1,6850^{*}$ & $1,5570^{*}$ & $1,4154^{\star}$ & $1,3186^{*}$ & $1,1885^{*}$ & $1,0215^{*}$ \\
\hline Control & 1,4928 & 1,2698 & 1,2013 & 0,9476 & 0,8378 & 0,7365 & 0,5825 \\
\hline
\end{tabular}

No yac. $=$ no racunados $(15)$

Vac.NR. = vacunados no tespondedores al PPD (16).

Vac. Re. = vacunados respondedores al PPD (16)

TBC $=$ pacientes con TBC activa (12).

Control = donantes de banco de sangre (12).

$* \mathbf{p}<0,01$.

a la luz de los nuevos métodos en uso desde hace algunos años.

No existen en la actualidad trabajos que muestren el impacto que tiene esta vacuna en la respuesta inmune. Ciertos autores han analizado, con técricas antiguas y pacientes poco seleccionados, algunos cambios inmunitarios posteriores a la vacuna BCG. Así, por ejemplo, midiendo la acción de la vacuna con una prueba de in hibición de la migración leucocitaria (MIF) en niños de 9 meses a 6 aftos, se obtuvieron resultados poco satisfactorios ${ }^{16}$. Otros no encontraron cotrelación entre la blastogénesis y la respuesta in vivo al "mantoux" en niffos de 22 meses de edad que habían sido vacunados al nacer ${ }^{17}$.

Nuestro estudio ha demostrado que, mediante un método para medir especificamente la respuesta de linfocitos $T$, es posible identificar modificaciones que diferencian a los vacunados y no vacunados. En efecto, en nifros sin BCG no encontramos casos con respuesta blastogénica y tampoco respuesta in vivo al PPD. Estos hallazgos contrastan con los comprobados en vacunados con reacciones positivas a la tuberculina, todos los cuales mostraron respuestas intensas in vitro, por encima de los valores extremos del grupo no vacunado. Es interesante resaltar que estos niños tienden a responder intensamente tanto a las concentraciones bajas de PPD como en las concentraciones elevadas del antígeno, lo que estaría respaldando la excelente capacidad de respuesta que produce el BCG en este grupo de lactantes.

Es importante hacer notar que las respuestas obtenidas en ambos grupos no estuvieron condicionadas a otros factores como la presencia de anticuerpos transferidos vía maternofetal o incapacidad funcional de este tipo de celulas. El estudio con PHA demostró excelente respuesta a este mitógeno. Consideramos también de gran importancia la ausencia de respuestas blastogé nicas y la anergia tuberculínica mostrada por los niffos no vacunados, pues estos resultados cuestionan afirmaciones recientes sobre la posibilidad de transferencia de inmunidad celular maternofetal a través de linfocitos aportados por la leche materna ${ }^{18}$.

En estudios anteriores habíamos determinado que existe en nuestra población de lactantes $7 \%$ de niños vacunados que no logran generar respuesta a repetidas aplicaciones de PPD. La presencia de cicatriz, el haber efectuado el estudio en forma prospectiva $y$ al azar, aseguraron fehacientemente que esto no se debía a errores de procedimiento, circunstancia invocada en algunos protocolos llevados a cabo con BCG. El estudio de estos niños permitiría, a nuestro entender, obtener datos de importancia para la comprensión de varias interrogantes que persisten en torno al uso de esta vacuna.

En efecto, del análisis particular de este grupo no respondedor se han caracterizado tres tipos de respuesta inmunitaria que podrían tener rele- 
vancia clínica. El primer tipo está constituido por 6 de los 16 niños $(37 \%)$, en quienes no hubo respuesta in vivo e in vitro al PPD, pero al ex. poner sus células a concentraciones altas del antígeno, éstas generaron respuestas en todo comparables a las de vacunados con PPD positivo. Estos lactantes podrian sufrir una falla en los mecanismos de presentación del antígeno, que se superaría incrementando la concentración del PPD. No sabemos aún si el de. fecto está ligado al período de inmadurez inmunológica que afecta a determinados recién nacidos o a otro nivel. Sin embargo, es posible que estos niños, con la estimulación permanente ambiental, pudieran transfomarse finalmente en PPD positivos. El segundo tipo de respuesta ocurrió en 9 de los 16 niños ( $56 \%$ ), en los cuales no se pesquisó respuesta inmunitatia in vivo y tampoco in vitro, este último estudio en concentraciones bajas y altas de PPD. Estos niños están siendo controlados periódicamente con el fin de revacunarlos al año de vida $y$, si continúan con respuestas negativas a estas pruebas, seguirlos a largo plazo con el objeto de determinar el impacto epidemiológico y la susceptibilidad que pudieran presentar al Mycobacterium tuberculosis. Este tipo de niños podría explicar los resultados contradictorios obtenidos en protocolos a largo plazo, en países como la India, donde no fue posible encontrat correlaciones con el uso de esta vacuna. El tercer tipo de respuesta estuvo representado por un solo riño $(7 \%)$ que mostró ausencia total de reacción in vivo e in vitro por un fenómeno de supresión activa de la respuesta inmune después de recibir la vacuna $\mathrm{BCG}$, inhibición que se demostró retirando de los cultivos las células con propiedades adherentes (monocitos), hallazgo de extraordinaria importancia, desde el mornento que estos nifios estarian con un riesgo potencial de hacer infecciones tuberculosas severas y graves. Esta observación no ha sido descrita en nifros vacunados, solamente en pacientes portadores de TBC, y permite postulat un defecto, probablemente genético, donde los determinantes antigénicos de estas mycobacterias activan a células con propiedades supresoras. Las características clínicas de estos pacientes han sido publicadas en extenso por Eliner, quien ha demostrado la gravedad de la enfermedad, así como la presencia de anergia cutánea y cierto grado de monocitosis periférica ${ }^{19}$. Ignoramos si a este tipo de personas pertenece parte de los casos graves de tuberculosis que aún se ven en pediatría. Lo importante es que están en curso investigaciones para extrapolar nuestras observaciones a este grupo que consideramos de un riesgo potencial muy severo.

La utilización de métodos serológicos para medir la inmunidad humoral desencadenada por el $M$. tuberculosis se ha desarrollado sólo en los últimos años. No hay antecedentes en la literatura sobre los cambios que ocurren en estos anticuerpos después de la vacunación con BCG, pero los resultados que hemos expuesto demuestran y confirman la escasa participación de la respuesta mediada por anticuerpos después de recibir la vacuna $B C G$ y la importancia de las poblaciones de células $\mathrm{T}$ en los fenómenos biológicos que acompañan a la infección por mycobacterias. La respuesta de anticuerpos no parece influir en la mayor o menor reactividad proliferativa de dichas células. En todo caso, parece necesario buscar métodos alternativos de análisis cuando se quieran investigar estas inmunoglobulinas.

En lo que respecta a las relaciones de identidad existentes en las reacciones producidas por PPD y BCG, investigaciones llevadas a cabo en los últimos años han demostrado que el $M$. tuberculosis comparte un antigeno proteico inmunorreactivo con el $M$. bovis ${ }^{20} ; \mathrm{y}$ un antígeno de 65 Kilodalton con una secuencia aminoacidica común se ha identificado en ambas mycobacterias y en el $M$. lepra. Por estos antecedentes ten íamos interés en comparar las respuestas inmunitarias producidas en pruebas de blastogénesis inducidas por PPD y BCG. Nuestros resultados sugieren que ambas respuestas son en todo semejantes. La identificación de este tipo de respuesta inmune a determinantes comunes en ciertas especies bacterianas será un tema de extraordinaria importancia en el futuro. Recordemos solamente que secuencias semejantes a la proteína 65 Kilodalton del $M$. tuberculosis se están identificando en bacterias gram negativas, lo que agrega nuevas interrogantes al rol que tienen estos microorganismos en la interpretación de la respuesta inmune al agente de la $t a-$ berculosis ${ }^{21}$.

La capacidad inmunitaria celular, observada en los lactantes de tres meses, vacunados y no vacunados, mediante la respuesta de las células $T$ a la estimulación con fitohemaglutinina, fue excelente, confirmando que ésta es normal en los lactantes menores. Por lo tanto, la mayor susceptibilidad de algunos nifios de esta edad a determinados patógenos obliga a buscar su expli- 
cación en defectos muy específicos, más que en alteraciones severas de la respuesta inmune. Estas observaciones explican, en parte, las dificultades que tenemos para individualizar las causas por las cuales algunos niffos presentan infecciones recurrentes.

\section{Resumen}

Para analizar la respuesta inmunitaria producida por la vacuna BCG se estudiaron lactantes de tres meses inmunizados al momento de nacer con reacción $\mathrm{PPD}$ positiva ( $\mathrm{n}: 16)$ y negativa ( $n$ : 16) y riños controles ( $n: 15$ ) de la misma edad no vacunados, mediante prueba específica de transformación blástica inducida por PPD en sus poblaciones de linfocitos $T$ y estimulación inespecífica de la mitosis con fitohemaglutinina. En no vacunados la respuesta blastogénica a concentraciones de 25,50 y $100 \mu \mathrm{g}$ de PPD fue escasa, 770 a $1700 \mathrm{cpm}$; en vacunados con PPD positivo $27925 \pm 7095$, $34297 \pm 8717$ y $45354 \pm 8453 \mathrm{cpm}$, respectivamente ( $p<0,001)$. En vacunados con PPD negativo fue similar a la respuesta de no vacunados en diez, mientras seis respondieton como los vacunados con reacción positiva, pero sólo a dosis de $100 \mu \mathrm{g}$ de PPD, con $22744 \pm 7145$ cpm $(\mathbf{p}>0,05)$. Los índices de estimulación obtenidos con PPD y BCG fueron del todo comparables. La respuesta a fitohemaglutinina fue $124945 \pm 53200 \mathrm{cpm}$ en no vacunados, 156263 \pm 43540 en vacunados PPD negativos $y$ $138217 \pm 22345$ en PPD positivos ( $p>0,05$ ). Los indices de estimulación con PPD, después de retirar las células con actividad supresora (monocitos), disminuyeron en todos los grupos, pero sólo en los vacunados no respondedores de manera significativa ( $p<0,01$ ). Las concentraciones séricas de anticuerpos $\lg \mathrm{G}$ e $\lg M$ anti. PPD fueron semejantes en todos los grupos, entre éstos y sueros provenientes de dadores sanos de un banco de sangre, pero significativamente menores que en los pacientes con tuberculosis activa. Existe buena correlación de las respuestas in vivo a la tuberculina y las pruebas in vitro en nifíos no vacunados y vacunados con reacción positiva a] PPD. Los niños no respondedores mostraron tres tipos distintos de respuesta y evidencia de ciertos mecanismos supresores. La excelente capacidad de respuesta $T$ a mitó- genos confirma la inmunocompetencia que existe a esta edad. EI BCG no produjo concentraciones séricas de anticuerpos anti-PPD medibles por ELISA. La semejanza de la respuesta in vitro al BCG y el PPD confirma la existencia de una homología antigenica entre ambos compuestos.

(Palabras claves: Vacuna BCG, inmunidad celular, humoral, linfocitos T.)

\section{Referencias}

l. Fine $P$ : BCG vaccination against tuberculosis and leprosy. Br Med Bull 1988; 44: 704-716.

2. WHO study Group: BCG vaccination policies. WHO tech Rep Ser 1980 . No 652 .

3. Ten Dam HG: Research on BCG vaccination. Adv Tuberc Res 1984; 21 : 79-106.

4. Tripathy SP: Estudio sobre la presencia por BCG en India. 15 años de seguimiento. Bol Union Int contra TBC y Enfemedades Respiratorias 1987 . 62: $65-69$

5. Sorensen $R$, González $B$. Sepülyeda $R$ : Aspectos intuunológicos de la vacunación BOG. Enf Resp Cit Torac $1990 ; 6: 32-37$.

6. Sepulveda $R$, Ferrer $X$, Sorensen $R$ : The effect of Bacillus Calmete Guerin immunization on the booster effect of tuberculin testing in healthy young adults. Am Rev Respir Dis 1989; 139: A 395 .

7. Scpulveda R, González B, Gerszencveig $R$, Ferrer $X$, Sorensen $R U$ : The influence of BCG immunization on tuberculin reactivity of healthy Chilean women in the third trimester of pregnancy. Am Rev Resp Dis (en prensa).

8. Nash $D R$, Douglas $J E$ : Anergy in active pulmonary tuberculosis: a comparison between positive and negative reactors and evaluation of 5 TU and 250 TU skin doses. Chest $1980 ; 17: 32-37$.

9. Danie! TM, Oxtoby MJ. Pinto $E$, Moreno $E$ : The inmune spectrum in patients with pulmonary tuberculosis. Am Rev Respir Dis 1981; 123 : 556 . 559.

10. Elliter $J$, Wallis $R$ : Immunologic Aspects of Mycobacterial infections. Rev Infect Dis 1989; 2: 5455-5459.

11. Boyum A: Separation of lymphocytes from blood and bone marrow. Introduction. Scand J Clin Lab Invest 1968; 7: 32-38.

12. Crowle Af. May M: Preliminary demonstration of tuberculo-immunity in vitro. Infect Immun 1981 ; $31: 453464$.

13. Greaves $M$, Janossy $G$, Doenhof $M$ : Selective triggering of human $\mathbf{T}$ and $\mathbf{B}$ lymphocytes in vitro. by polyclonal mitogens. J Exp Med 1974; 140: 1-8.

14. Inostroza $J$, Schneider $I$, Soza $G$, Castillo $H$, Ossa $A$ : Detection of antibodies to Protein Purifies (PPD) for early diagnosis of tuberculous Meningitis. Praxis und Klinik der Pneumologie 1986; 40: $94-95$ 
15. Swinscow TDV: Statistics at Square One. Pub British Medical Association. Fourth edition. 1978.

16. Seth $V$, Kukreka $N$, Bentra A: Immunological aspects of BCG. J Tropical Pediatrics 1984; 30 : $210-216$.

17. Grindutis H, Baynham MLO, Scott PH: Arch Dis Child 1984; $59: 614-617$.

18. Keller MA, Rodriguez AL, Atvarez $S$, wheeler $N C$ : Transfer of tuberculin immunity from mother to infant. Pediat Res 1987; 22: 277-281.

19. Ellner $f J$ : Suppressor adherent cells in human tuberculosis. J Immunol 1978; 121 : 2573-2579.

20. Shinnick TM: The 65 Kilodalton antigen of $M y$. cobacterium tuberculosis. J Bacteriol 1987; 169: 1080-1088.

21. Snider D: Research towards global control and prevention of tuberculosis with an emphasis on vaccine development. Rev Infect Dis 1989; 2: $5335-5490$. 